\title{
Transportation of long objects in unknown cluttered environments by a team of robots: a dynamical systems approach
}

\author{
Toni Machado, Tiago Malheiro, Sérgio Monteiro and Estela Bicho \\ Department of Industrial Electronics \\ University of Minho \\ 4800-058 Guimarães, Portugal \\ Emails: \{tmachado, tmalheiro, sergio, estela\}@dei.uminho.pt
}

\author{
Wolfram Erlhagen \\ Department of Mathematics and Applications \\ University of Minho \\ 4800-058 Guimarães, Portugal \\ Email: wolfram.erlhagen@math.uminho.pt
}

\begin{abstract}
We present a distributed architecture for teams of two autonomous mobile robots that act in coordination in a joint transportation task of long objects. The team is able to perform its transportation task in unknown environments while avoiding static or moving obstacles. The working environment can be cluttered and with narrow passages such as corridors, corners and doors. These characteristics make our approach suitable to be deployed in warehouses or office-like environments. The control architecture of each robot is formalized as a non-linear dynamical system, where by design attractor states dominate. The overt behavior is smooth and stable, because it is generated as a time sequence of attractor states, for the control variables, which contributes to the overall asymptotically stability of the system that makes it robust against perturbations. We present results with real robots in a real indoor cluttered environment.
\end{abstract}

Index Terms-multi-robot system, joint transportation, autonomous mobile robots, dynamical system.

\section{INTRODUCTION}

The transportation of a specific object/payload from point A (initial location) to point B (destination location) is a requirement in a large number of scenarios. Some examples of these scenarios include warehouses, depots, ports, construction sites, and industrial processes. When the objects to be transported are of large dimensions, instead of using single large robots, it is more efficient the use of teams of robots that act in coordination in a joint action transportation task of the payload to its destiny. This problem is aggravated if the environment is too cluttered or if it presents narrow corridors and corners.

In this context, experiments with automated guided vehicles are reported in [6] and [9]. In [10] two robots coordinate to transport a long payload, using a leader-follower strategy. The purpose is to deploy the team in Mars. In [1] two robots transport a long ladder in a corridor with a $90^{\circ}$ corner (but only in simulation). In [4] authors present a simulation of two robots manipulators carrying large objects within a multi-agent architecture.

In previous work we have presented control architectures for teams of mobile robots with the capacity to transport objects in cooperation [8], but had some limitations on cluttered environments. Here, we present a new architecture tailored for

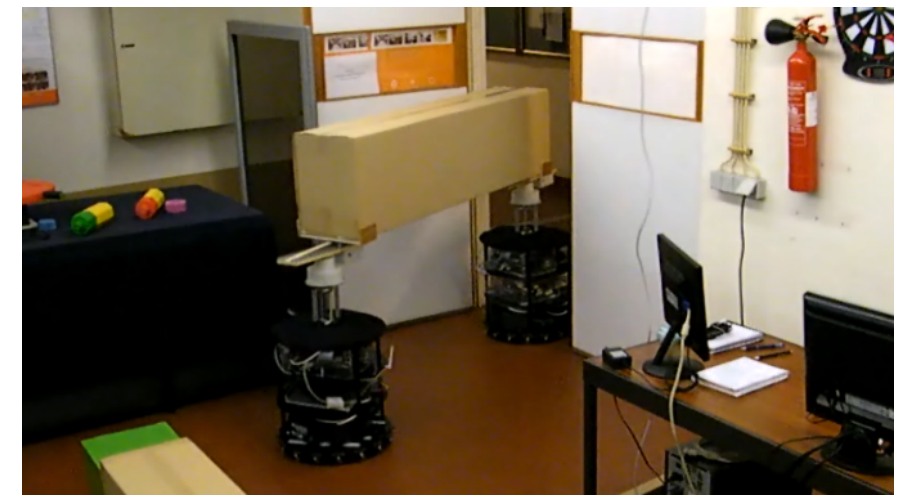

Fig. 1. The team of robots transporting a long box as payload.

teams of two robots that transport long objects in cooperation (see Fig. 1) and must be able to navigate in narrow corridors with corners. Opposite to some of the previous related work, no reference trajectories are computed nor communicated to the follower (as opposed to [1], [7], [11], for instance). The ability to avoid obstacles is provided for either static or moving obstacles (as opposed to [10]).

In this paper we present a control architecture where the helper (rear end robot) will steer such that it tries to make the transported object align with the heading direction of the leader (front robot). The communication between the two robots is minimal. Only the angle that the payload makes with the leader's heading direction is communicated from this robot to the helper robot.

The control architecture is formalized using the attractor dynamics approach to behavior based robotics [2], [3], in the form of behaviors for obstacle avoidance and object transportation. The overt behavior is smooth because the movement of each robot is generated by a time series of attractor (i.e. asymptotically stable) states, which implies that there is always an exponential relaxation to a stable equilibrium point.

The remainder of the paper is structured as follows: section II presents the robots and the problem to be tackled; then, in section III we define the behavioral dynamics for the helper 


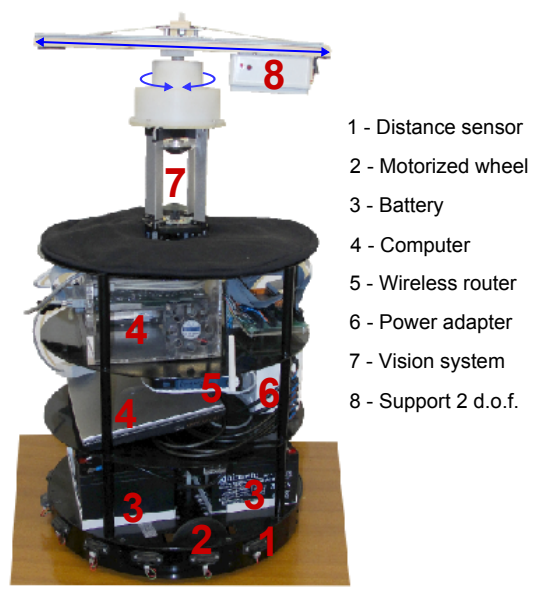

Fig. 2. Photo of one of the robots used in the experiments.

robot; after, some results are shown in section IV; finally, we end with conclusions and future work.

\section{ROBOT TEAM AND TASK CONSTRAINTS}

The team is composed by two autonomous mobile robots equal to the one depicted in Fig. 2. Each robot is built by: i) two differential motorized wheels to drive it; ii) two caster wheels to keep it balanced; iii) eleven infrared distance sensors to detect the obstructions on its navigation path; iv) one wireless router to enable remote access in order to facilitate the configuration and communication between them; v) one omni-directional vision system to acquire targets; vi) batteries and a power adapter box to supply power to all the hardware; and vii) a dedicated support to hold the payload to transport, that is instrumented such that it provides information about the displacement of the payload with respect to the center of the robot, and its relative bearing (see Fig. 3). The maximum displacement of the payload is $20 \mathrm{~cm}$ to each side of the robot's center. The leader has two modest computers (one is a Via $1.0 \mathrm{GHz}$ and the other is a Centrino $1.5 \mathrm{GHz}$ ) to access all the hardware and control the robot's behavior. Since the helper does not use the vision system, it only has one computer (Via $1.0 \mathrm{GHz}$ ).

The mission of the team is to transport an object from a starting location (payload loading location) to a destination location (payload unloading). Both the loading and unloading actions are not subject of the present study in this paper. The team is composed by two robots: one leader and one helper. The leader acquires targets information, i.e. where the loading and unloading locations are, by means of its vision system, and leads the team from one place to the other. When moving, the leader broadcasts its bearing direction, $\theta_{L}$, to the helper. The helper uses this information, together with the data obtained from its payload support base (bearing of the payload $\theta_{H}$ and displacements $d_{H}$ of the payload with respect to the center of the robot) and low-level obstacle sensors, to compute its linear and angular velocities. In order to avoid payload collision with obstacles, it is assumed that the payload to transport should not be larger than the robots' diameter and

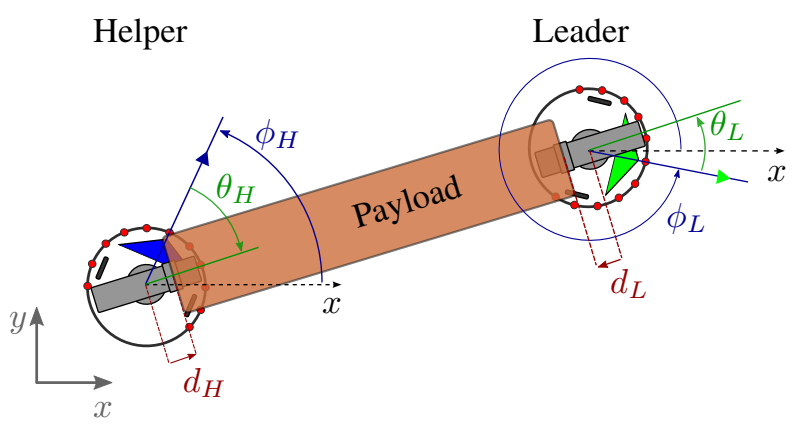

Fig. 3. The leader's and helper's heading direction, $\phi_{L}$ and $\phi_{H}$, respectively, are measured relatively to an arbitrary, but fixed, external reference frame (to obtain these angles should be considered that the reference frame moves with each robot's center but its rotation is locked, as in [2]). Each payload support base is instrumented such that it can measure the displacement of the payload with respect to the center of the robot, $d_{L}$ or $d_{H}$, and the bearing of the payload, $\theta_{L}$ or $\theta_{H}$, i.e. the angle that the payload makes with the current heading direction of the leader and helper robot, respectively.

its length should be smaller than two times the distance range of the obstacle sensors. The range of the obstacle sensors is a parameter that can be set as a function of the payload length. However, as will be seen in the next section this parameter does not explicitly enter into the control systems that governs the robots' behavior. Only the displacement of the payload with respect to robots' centers, are used and these are an output of the payload support base.

\section{THE DYNAMICS OF COORDINATED MOTION}

To model each robot behavior we use, as control variables, the heading direction, $\phi_{r}$, with respect to an arbitrary but fixed reference frame, and the path velocity, $v_{r}(r=\{L, H\}$, where $L$ means leader and $H$ means helper). Behavior is generated by providing values to these variables, which are obtained from constant solutions of dynamical systems where, by design, attractor solutions (i.e. asymptotically stable states) dominate. The behavioral dynamics of $\phi_{r}(t)$ and $v_{r}(t)$ are governed by differential equations:

$$
\begin{gathered}
\frac{d \phi_{r}(t)}{d t}=f_{d e s, r}\left(\phi_{r}(t)\right)+f_{o b s, r}\left(\phi_{r}(t)\right)+f_{\text {stoch }, r} \\
\frac{d v_{r}(t)}{d t}=-\lambda_{v, r}\left(v_{r}(t)-v_{d e s, r}\right) \quad, \quad \lambda_{v, r}>0
\end{gathered}
$$

where $f_{\text {des }, r}\left(\phi_{r}(t)\right)$ models a target acquisition behavior by dynamically orienting the robot to a desired (target) direction, $f_{o b s, r}\left(\phi_{L}(t)\right)$ models the obstacle avoidance behavior, and $f_{\text {stoch, } r}$ is a stochastic force, that ensures escape from unstable equilibrium states, when bifurcations take place (i.e. an attractor becomes a repeller) and the system sits in one. Eq.(2) represents a linear dynamical system that attracts the linear velocity to the value specified by $v_{d e s, r}$, with a relaxation rate defined by $\lambda_{v, r}$.

The leader's behavioral dynamics has been previously defined and evaluated (see [2] for details).

Next, we explain for the helper robot how the individual contributions to vector fields in (1) and (2) are built. 
As a design strategy, the helper should steer in order to keep the payload aligned with the leader's heading direction, i.e. to be exactly behind the leader, thus bringing $\theta_{L}$ to zero (see Fig. 3). Furthermore, the helper's path velocity is adjusted so that the distance between the two robots is kept approximately constant.

\section{A. Attractor dynamics for the helper's heading direction}

As seen above, the heading direction dynamics, (1), has two main contributions, which for the helper robot mean: 1) $f_{\text {des }, H}\left(\phi_{H}(t)\right)$ is a transport formation contribution, responsible to orient the helper to a direction that allows it to rapidly align the payload with the leader's heading direction; 2) $f_{o b s, H}\left(\phi_{H}(t)\right)$ is an obstacle avoidance contribution that repels its heading direction from undesired values, i.e. the location of sensed obstructions. Next, we present these two contributions.

1) Transport formation contribution: $f_{d e s, H}\left(\phi_{H}(t)\right)$ is defined by:

$$
f_{\text {des }, H}\left(\phi_{H}(t)\right)=-\lambda_{\operatorname{trp}, H} \sin \left(\phi_{H}(t)-\psi_{\text {des }, H}\right)
$$

which erects an attractor at the desired heading direction $\psi_{\text {des, } H}$ for the helper, with a relaxation rate defined by $\lambda_{t r p, H}>0$, and a repeller in the opposite direction.

The desired heading direction $\psi_{\text {des }, H}$ is the one that puts the leader exactly behind the leader, thus making the payload aligned with the leader's heading direction. It is important to do it in this way, because then the team actually navigates around the corners, instead of cutting through them and colliding with corner walls. It can be computed from:

$$
\psi_{\text {des }, H}=\phi_{H}+\theta_{H}+\gamma_{\theta, L}
$$

where $\phi_{H}$ is is the helper's current heading direction, $\theta_{H}$ is the angle measured by the helper's own payload support, i.e it is the angle on which the helper senses the movement of the payload, and $\gamma_{\theta, L}$ contributes to align the payload with the leader. It is defined by:

$$
\gamma_{\theta, L}= \begin{cases}\gamma_{f a c} \lambda_{\theta_{L}}\left(\frac{2}{1+\exp \left(\beta_{\theta_{L}} \theta_{L}\right)}-1\right) & , \Delta L>0 \\ \theta_{L} & , \text { otherwise }\end{cases}
$$

where $\lambda_{\theta_{L}}$ limits the helper's turning angle (here set equal to $\pi / 2) ; \theta_{L}$ is the angle that the payload makes to the current leader's heading direction, as measured by the leader's payload support base (see Fig. 3) and communicated to the helper; $\beta_{\theta_{L}}$ is a parameter used to tune the desired helper's curvature radius to align the payload with the leader's heading direction; $\gamma_{f a c}$ is used to reduce the value of the desired curvature rate such that the helper does not drop the object, giving priority to recover the payload support's displacement over of the task to align the payload with the leader's heading direction. It reads:

$$
\gamma_{f a c}=1-\left|d_{H} / d_{H, \max }\right|^{n} .
$$

Here, $d_{H}$ is the displacement of the payload with respect to the center of the helper, and is measured by the helper's own payload support base (see Fig. 3), and $d_{H, \max }$ is the maximum allowed displacement $(20 \mathrm{~cm})$. The parameter $n$ is used to adjust the decay rate of $\gamma_{f a c}$ (in our implementation $n=3$ ).

$\Delta L$ in (5) signals if the leader is navigating in a direction that tends to increase the distance between robots $(\Delta L=1)$ or not $(\Delta L=-1)$ and it is defined by:

$$
\Delta L=\left\{\begin{array}{rl}
1 & , \quad-\pi / 2 \leq \theta_{L}<\pi / 2 \\
-1 & , \quad \text { otherwise }
\end{array}, \forall \theta_{L} \in[-\pi, \pi]\right.
$$

Finally, note that if we replace (4) into (3) one gets:

$$
f_{\text {des }, H}\left(\phi_{H}(t)\right)=\lambda_{t r p, H} \sin \left(\theta_{H}+\gamma_{\theta, L}\right)
$$

which means that in the implementation there is no need to maintain an estimate of the helper's heading direction. Thus, making the system immune to calibration and odometry errors.

2) Obstacle avoidance contribution: The obstacle avoidance contribution is responsible to ensure collision avoidance with sensed obstructions and it is defined by:

$$
f_{\text {obs }, H}\left(\phi_{H}(t)\right)=\sum_{i=1}^{n \text { Sectors }} f_{o b s, i, H}\left(\phi_{H}(t)\right)
$$

where $n$ Sectors is the number of distance sensor sectors that the robot has, and $f_{o b s, i, H}\left(\phi_{H}(t)\right)$ represents the contribution of the distance sensor in sector $i$. Each of this contributions is defined by:

$$
\begin{aligned}
f_{o b s, i, H}\left(\phi_{H}(t)\right)= & \lambda_{o b s, i}\left(\phi_{H}(t)-\psi_{o b s, i}\right) \times \\
& \times \exp \left(-\frac{\left(\phi_{H}(t)-\psi_{o b s, i}\right)^{2}}{2 \sigma_{i}{ }^{2}}\right)
\end{aligned}
$$

which puts a repeller at direction $\psi_{o b s, i}$, with strength of repulsion defined by $\lambda_{o b s, i}>0$, and with $\sigma_{i}$ setting the angular range over which the repeller exerts its repulsive force (see [2] for details).

In principle a robot that detects an obstruction on a particular direction should move away from that direction. Here, because the team is linked by an object that they transport cooperatively, then the presence of that object must also be accounted for. Thus, the direction at which each repeller $i$ is erected is made:

$$
\psi_{o b s, i}=\phi_{H}+\Psi_{o b s, i}
$$

where $\Psi_{o b s, i}$, accounts for the fact that a payload is being carried with a bearing angle $\theta_{H}$, and is defined by:

$$
\begin{gathered}
\Psi_{o b s, i}= \begin{cases}-\delta & ,\left(\theta_{H} \geq 0\right) \wedge\left(0 \leq \varrho_{i} \delta \leq \theta_{H}\right) \\
+\delta & ,\left(\theta_{H}<0\right) \wedge\left(\theta_{H} \leq \varrho_{i} \delta \leq 0\right) \\
\varrho_{i} \delta & , \text { otherwise }\end{cases} \\
, \forall \theta_{H} \in[-\pi, \pi] \wedge \forall i \in \mathbb{N}: i \leq n \text { Sectors }
\end{gathered}
$$

Here $\delta$ represents the angular distance between the center of two consecutive distance sensors and $\varrho_{i}=\{-5,-4, \ldots, 4,5\}$ is the sector position with respect with the robot's heading direction (see Fig. 4b).

Fig. 4a shows one example scenario, where the helper senses an obstruction on its left side, which must avoid by 


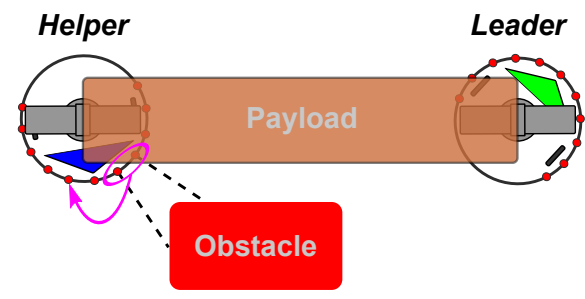

(a) Obstacle contribution shifted

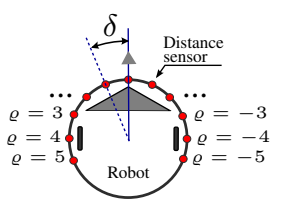

(b) Positions of the distance sensors

Fig. 4. Obstacles detected between helper's heading direction and payload movement direction are moved to a strategic position, in order to avoid payload collision with obstacles.

turning counterclockwise, i.e. towards the obstacle, because the object and the leader are also on that side of the obstacle.

Finally, as in (8), note that if we replace (11) into (10) one gets:

$$
f_{o b s, i, H}(\phi(t))=-\lambda_{o b s, i} \times \Psi_{o b s, i} \times \exp \left(-\frac{\Psi_{o b s, i}{ }^{2}}{2 \sigma_{i}{ }^{2}}\right)
$$

which means, again, that in the implementation there is no need to maintain an estimate of the helper's heading direction.

\section{B. Desired path velocity for the helper robot}

We have seen that the path velocity dynamics, in (2), erects an attractor in the desired value $v_{d e s, H}$ for the helper's path velocity. This value must be set so that the helper adjusts its path velocity trying to bring $d_{H}=0$ (by design of the payload support, the displacement of the slider joint is equal on both robots). For that we use a PID controller to set the desired path velocity:

$$
v_{d e s, H}=\Delta H\left(k_{p} d_{H}(t)+k_{i} \int_{0}^{t} d_{H}(\tau) d \tau+k_{d} \frac{d d_{H}(t)}{d t}\right)
$$

where $k_{p}, k_{i}$ and $k_{d}$ are, respectively, the proportional, integral and derivative controller gains, and $d_{H}(t)$ is the support's displacement from the robot's center. The integral and differential terms compensate for the fact that of the helper does not have information about the leader's linear velocity. Similarly to $\Delta L, \Delta H$ signals if the helper is navigating in a direction that approaches $(\Delta H=1)$ or not $(\Delta H=-1)$ the leader, and it is given by:

$$
\Delta H=\left\{\begin{array}{rl}
1 & -\pi / 2 \leq \theta_{H} \leq \pi / 2 \\
-1 & \text { otherwise }
\end{array}, \forall \theta_{H} \in[-\pi, \pi]\right.
$$

\section{RESUlts}

Here we present an experiment with real robots conducted in an office like environment with tight spaces to maneuver around, where the robotic team transports a payload that is $1.5 \mathrm{~m}$ long. The layout is depicted in Fig. 5, where it can also be seen the initial and final locations, the intermediate targets and the cameras points of view. The destination of the team is the blue box (Target 3). The intermediate targets (Target 1 and 2) are used by the leader and are only required to make the team navigate that specific cluttered path.

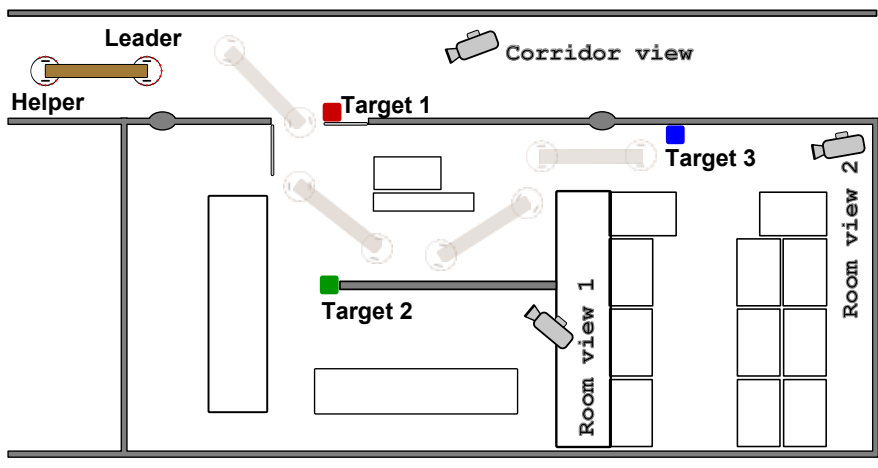

Fig. 5. Layout of the environment where the experiment was conducted. The locations of the targets and cameras view are also signalized.

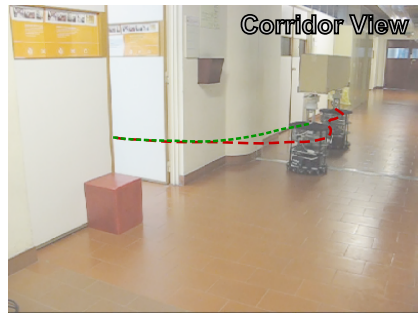

(a)

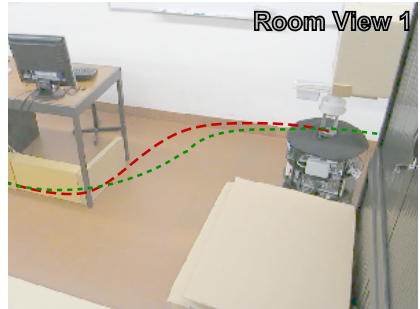

(c)

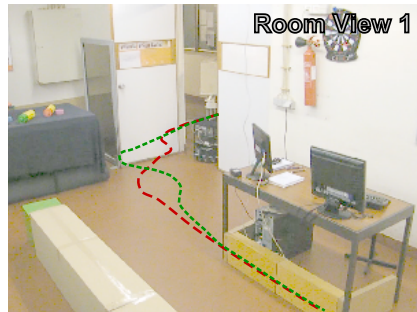

(b)

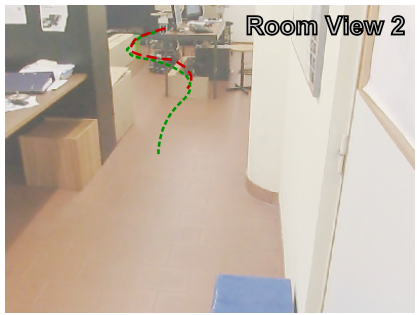

(d)
Fig. 6. Video snapshots of an experiment, where the robotic team transports a box as payload. The dashed lines represent the robots' paths: leader's green (small dashed) line; helper's - red (long dashed) line.

In Fig. 6 the robots' paths are represented from each of the cameras' viewpoints. The result shows that helper has a larger curvature radius than its leader in order to avoid payload collision with obstacles while at the same times the helper tries to align the payload with the leader's heading direction.

Some snapshots are presented with the corresponding internal dynamics in Fig. 7. The team is able to successfully surpass all the corners to its destination.

Four moments of the experiment are described here. The first one is when the team is passing through the door, Fig. 7a. At that moment, due the leader is too close to the target 2 it changes its target to the final destination (target 3), as can be seen in Fig. 7b. The helper's transport formation contribution is trying to orient it to its right but the obstacle contribution forces it to avoid collision with the opened door (Fig. 7c). In the second moment (Fig. 7d) the helper is steering clockwise to align the payload with the leader's heading direction (see Fig. 7e and Fig. 7f). The leader is trying to align its heading 


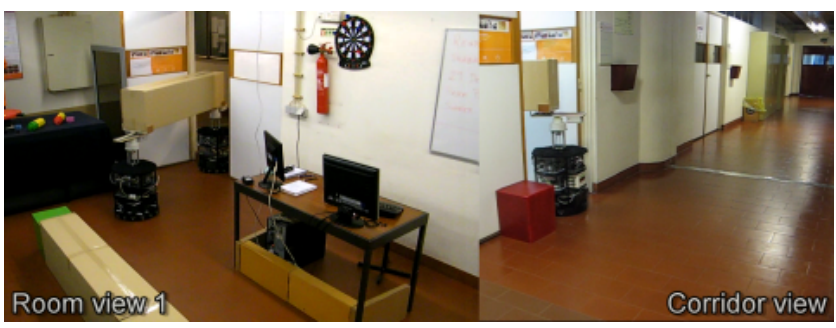

(a) Two camera views at time $=29 \mathrm{~s}$

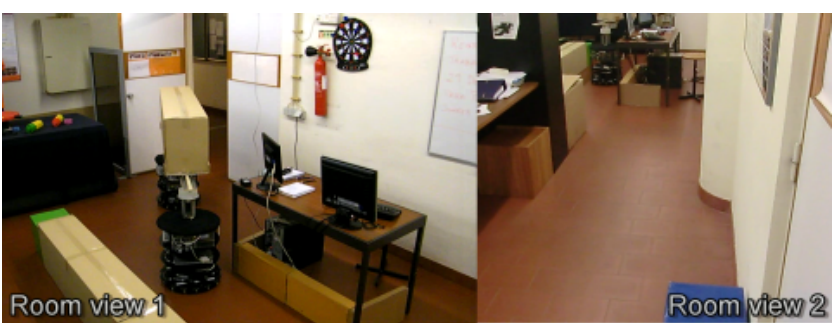

(d) Two camera views at time $=43 \mathrm{~s}$

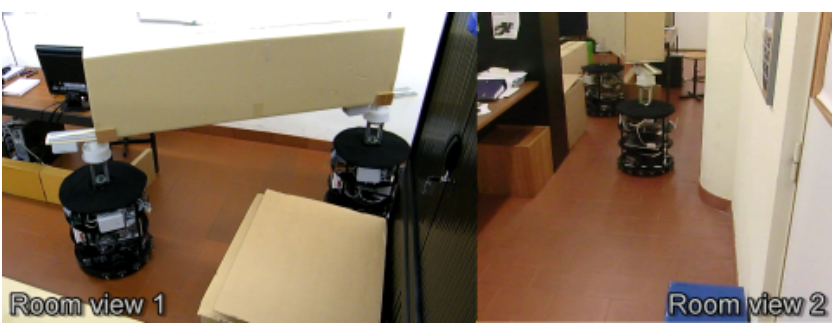

(g) Two camera views at time $=68 \mathrm{~s}$

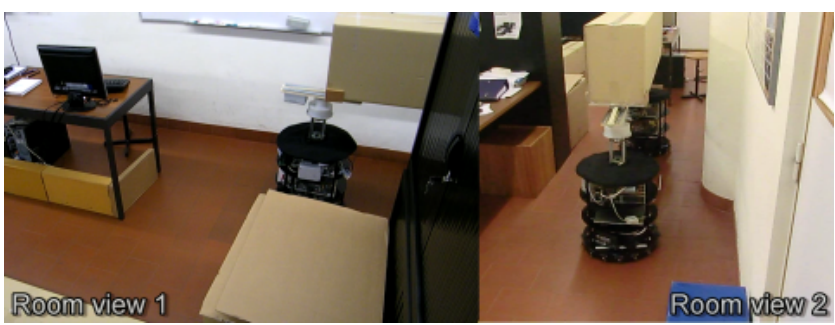

(j) Two camera views at time $=77 \mathrm{~s}$

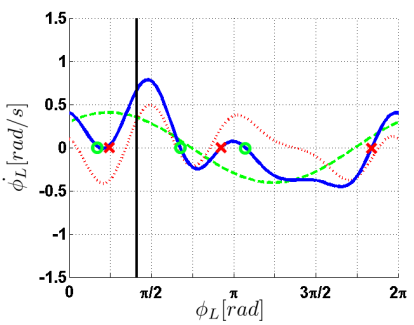

(b) Leader dynamics at time $=29 \mathrm{~s}$

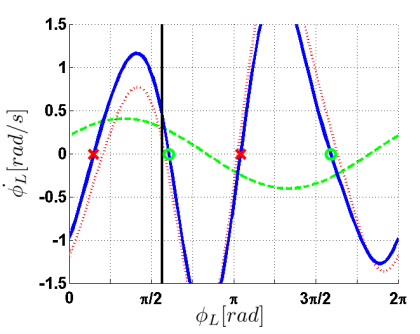

(e) Leader dynamics at time $=43 \mathrm{~s}$

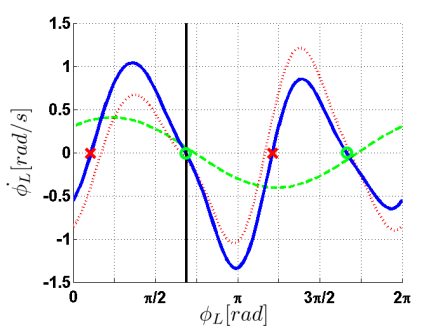

(h) Leader dynamics at time $=68 \mathrm{~s}$

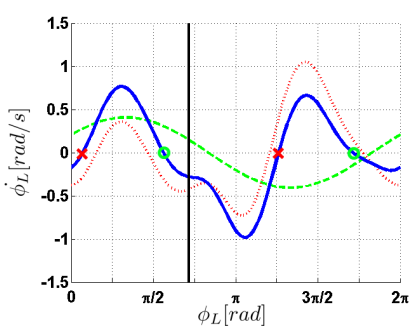

(k) Leader dynamics at time $=77 \mathrm{~s}$

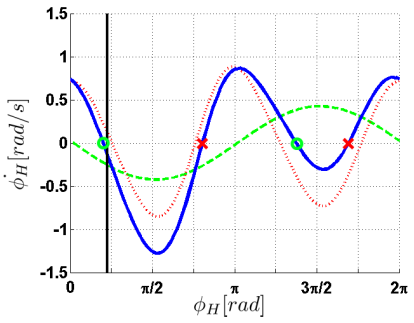

(c) Helper dynamics at time $=29 \mathrm{~s}$

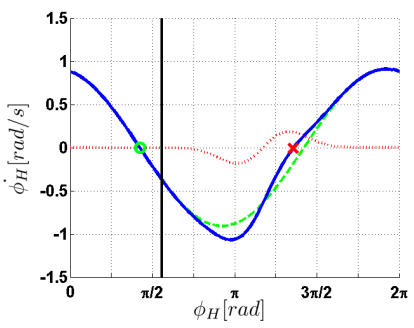

(f) Helper dynamics at time $=43 \mathrm{~s}$

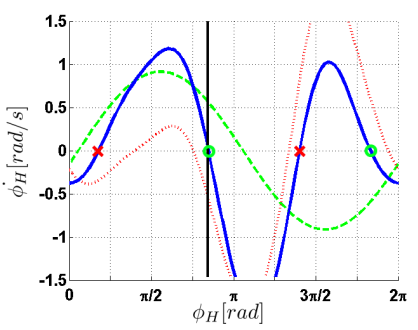

(i) Helper dynamics at time $=68 \mathrm{~s}$

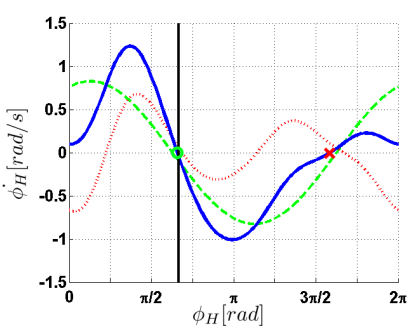

(1) Helper dynamics at time $=77 \mathrm{~s}$

Fig. 7. Snapshots of the experiment in Fig. 6. The left column shows the team of robots transporting the payload in the environment from two cameras' viewpoints. The center image (left column of plots) and the rightmost show the corresponding leader's and helper's dynamics, respectively. Legend of the plots: green (long dashed) line - target contribution $f_{d e s, r}\left(\phi_{r}(t)\right)$; red (small dashed) line - obstacles contribution, $f_{\text {obs }, r}\left(\phi_{r}(t)\right)$; blue (solid) line - result contribution, (1); vertical solid line represents the robot's heading direction, $\phi_{r}$; and green circles and red crosses highlight the attractors and repellers of the result contribution. The full video about this experiment can be seen on http://marl.dei.uminho.pt/public/videos/ISIE2013.html.

direction to the target 3 but the obstacle contribution from both sides forces it to go ahead between the desk and the obstacles at its left, see Fig. 7e. In the third instant of time presented (see Fig. $7 \mathrm{~g}$ ) it is the most difficult part of this task, since the team has to pass in two consecutive corners $\left(90^{\circ}\right.$ to their left then $90^{\circ}$ to their right). In Fig. $7 \mathrm{i}$ can be seen that the transport formation contribution is trying to orient the helper to its left to avoid payload collision with the obstacle detected at its right, but the obstacle contribution does not allow it to rotate more in order to avoid collision with the desk. In the last moment (see Fig. 7j) the team goes ahead, stopping in front of the position of its destination (target 3 ). The internal dynamics of the leader shows that it is trying to orient itself to the target 3 , but the obstacle contribution due to the presence of the wall at its left does not allow to do it, they navigate parallel to the wall. The internal dynamics of the helper (see Fig. 71) shows that it is navigating in the direction specified by the transport formation contribution and it is the same direction imposed by the obstacle contribution, thus both contributions are forcing the helper navigating in front, as desired for this situation.

Fig. 8 shows the evolution of the fixed points of the heading dynamics together with the heading direction of the robots for the experiment presented in Fig. 7. These show that the system 


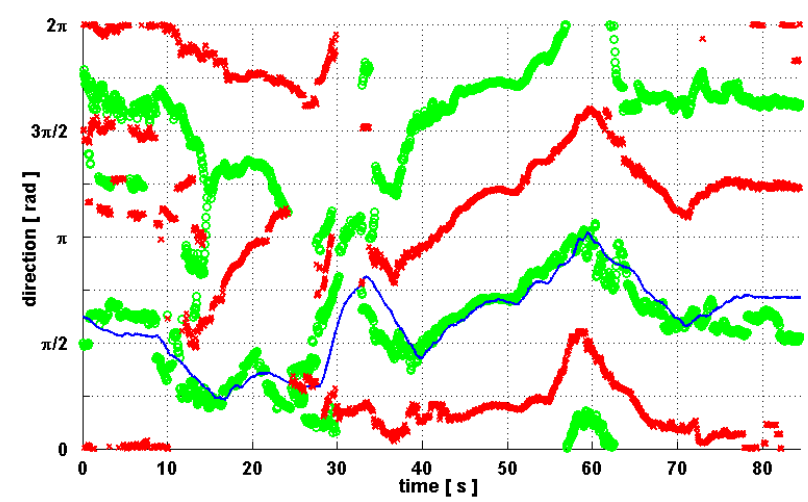

(a) Leader robot

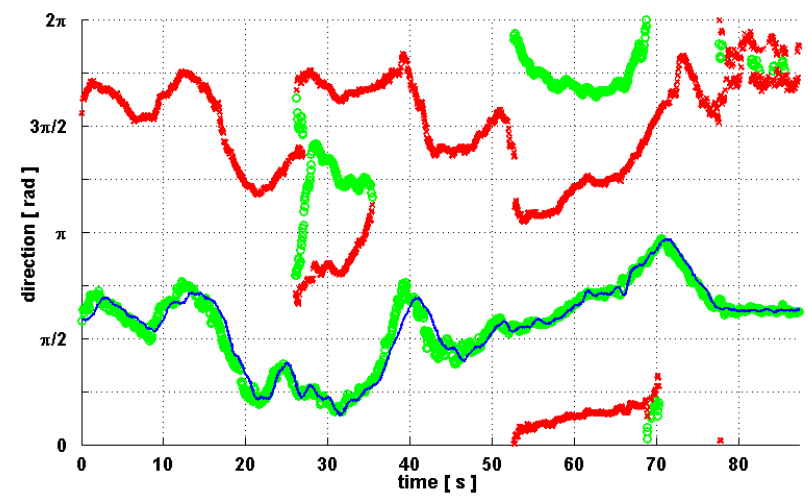

(b) Helper robot

Fig. 8. Temporal evolution of the fixed points of the heading direction and the corresponding heading direction for the experiment presented. Legend: green (circle) - attractors; red (cross) - repellers; blue (line) - robot's heading direction.

is well tuned, since the heading direction of each robot is able to track one of its attractors'.

The parameters used for the leader were: $\lambda_{t a r}=1 /(50 d t)$, $\beta_{1}=1 /(5 d t), \beta_{2}=0.3$, and $k_{v}=0.2$. The parameters used for the helper were: $\lambda_{t r p, H}=1 /(21 d t), \beta_{1}=1 /(4 d t), \beta_{2}=$ $0.3, k_{v}=0.3, k_{p}=2.5, k_{i}=0.9$ and $k_{d}=0.25$. The parameters $\beta_{1}$ and $\beta_{2}$ are used to tune the parameters $\lambda_{o b s, r}$ (see [2] for detail). For both robots leader and helper the integration time step was around $50 \mathrm{~ms}$, i.e. $d t \cong 0.05$.

\section{CONCLUSION AND FUTURE WORK}

In this paper we have presented a control architecture that allows a team of two autonomous mobile robots to engage in a joint transportation task.

As a design strategy, the helper tries to align the transported payload with the heading direction of the leader. The benefits of using this strategy are a better navigation in cluttered environments or with narrow corridors, such as storage spaces and warehouses. Further, the architecture performs in unknown and dynamic environments.

An experiment with real robots has been presented to validate the features of the architecture.

By using the attractor dynamics approach one also benefits from its advantages, showing smooth and stable trajectories even around obstacle courses.
In future work this architecture will be further improved. The next step is to remove the requirement of the leader to communicate its heading direction to the helper. This will be accomplished by implementing Dynamic Neural Fields [5] in the helper that allow to estimate and represent the attitude of the leader, for that it will be needed to develop a dedicated vision system on the helper robot. We intent to extend this control architecture to more than two robots, in order to transport other kinds of payload shapes.

\section{ACKNOWLEDGMENT}

This work is also supported by FEDER Funds through Competitivity Factors Operational Program - COMPETE and National Funds by FCT Portuguese Science and Technology Foundation under the Project FCOMP-01-0124-FEDER022674. We would like to thank all the people that work in our laboratory, MARL (Mobile and Anthropomorphic Robotics Laboratory) at University of Minho. All of them contributed in several ways for the success of this work. Toni Machado would also like to thank the Portuguese Science and Technology Foundation for providing his Ph.D. scholarship (ref. SFRH/BD/38885/2007).

\section{REFERENCES}

[1] Y. Asahiro, E. Chang, A. Mali, I. Suzuki, and M. Yamashita, "A distributed ladder transportation algorithm for two robots in a corridor," in Proc. of the Intl. Conf. in Robotics and Automation, ICRA 2001, 2001, pp. 3016-3021.

[2] E. Bicho, P. Mallet, and G. Schöner, "Target representation on an autonomous vehicle with low-level sensors," The International Journal of Robotics Research, no. 19, pp. 424-447, 2000.

[3] E. Bicho and G. Schöner, "The dynamic approach to autonomous robotics demonstrated on a low-level vehicle platform," Robotics and Autonomous Systems, no. 21, pp. 23-35, 1997.

[4] S. Djebrani and F. Abdessemed, "Multi-agent prototyping for a cooperative carrying task," in Proc. of the 2009 IEEE Intl. Conf. on Robotics and Biomimetics, Guilin, China, December 19-23 2009, pp. 1421-1426.

[5] W. Erlhagen and E. Bicho, "The dynamic neural field approach to cognitive robotics," Journal of Neural Engineering, vol. 3, pp. 36-54, September 2006.

[6] M. Hashimoto, F. Oba, and S. Zenitani, "Coordinative object - transportation by multiple industrial mobile robots using coupler with mechanical compliance," in Proc of the Intl. Conf. on Industrial Electronics, Control and Instrumentation, Maui, USA, 15-19 November 1993, pp. 1577-1582.

[7] Y. Kim and M. Minor, "Coordinated kinematic control of compliantly coupled multirobot systems in an array format," IEEE Transactions on Robotics, vol. 26, no. 1, pp. 173-180, February 2010.

[8] R. Soares, E. Bicho, T. Machado, and W. Erlhagen, "Object transportation by multiple mobile robots controlled by attractor dynamics: theory and implementation," in Proc. of the IEEE/RSJ Intl. Conf. on Intelligent Robots and Systems, San Diego, CA, Oct. 29 2007-Nov 2007, pp. 937944.

[9] B. Stouten and A. Graaf, "Cooperative transportation of a large object development of an industrial application," in Proc. Of the Intl Conf on Robotics and Automation, 2004, pp. 2450-2455.

[10] A. Trebi-Ollennu, H. Nayar, H. Aghazarian, A. Ganino, P. Pirjanian, B. Kennedy, T. Huntsberger, and P. Schenker, "Mars rover pair cooperatively transporting a long payload," in Proc. of the IEEE International Conference on Robotics and Automation, vol. 3, 2002, pp. 3136-3141.

[11] A. Yufka, O. Parlaktuna, and M. Ozkan, "Formation-based cooperative transportation by a group of non-holonomic mobile robots," in 2010 IEEE International Conference on Systems Man and Cybernetics (SMC), Istanbul, Turkey, 10-13 Oct. 2010, pp. 3300-3307. 